\title{
Estimativa do campo regional e residual gravimétrico a partir da aplicação de filtragem conjunta a dados sintéticos usando os métodos SVD e PCA
}

João Felipe de S. Ribeiro(IGEO-UFBA) \& Joelson da Conceição Batista(IGEO-UFBA) \& Alexandro G. Cerqueira(IGEO-UFBA)

Copyright 2018, SBGf - Sociedade Brasileira de Geofísica

Este texto foi preparado para a apresentação no VIII Simpósio Brasileiro de Geofísica, Salinópolis, 18 a 20 de setembro de 2018. Seu conteúdo foi revisado pelo Comitê Técnico do VIII SimBGf, mas não necessariamente representa a opinião da SBGf ou de seus associados. É proibida a reprodução total ou parcial deste material para propósitos comerciais sem prévia autorização da SBGf.

\section{Resumo}

The techiniques singular value decomposition (SVD), principal component analyses (PCA) and robust polynomial (PR) has been combined and tested in synthetic gravity anomalies for delineation of subsurface features. The comparative analysis of the results was performed considering data with and without random noise addition, which allowed a study of the noise elimination capacity using the PCA and SVD techniques, and along with the PR, accomplish a association between these methods for differentiates the regional and local anomalies generated by the proposed synthetic model. In this paper was possible to study and compare the results allowing to understand the advantages and disadvantages of methods in the elimination of influence of the noise and in separation regional and local anomalies. The entire generation, filtering and parsing procedure has been implemented in the Python language and its libraries are open to the public.

\section{Introdução}

A separação dos efeitos do campo gravimétrico produzido pela superposição de várias fontes distribuídas em subsuperfície é de difícil individualização (Beltrão et al., 1991). Os termos regional e residual são usualmente utilizados para diferenciar anomalias produzidas por massas ou corpos maiores, localizados a grandes profundidades, de anomalias locais cujas massas estão mais próximas da superfície. A definição de anomalia de campo regional e residual também poderá mudar de acordo com a profundidade do alvo a ser interpretado.

Ao logo do tempo foram propostos inúmeros métodos para a separação dos campos regional e residual (Jacobsen B. H., 1987; Mickus K. L. et al., 1991 e Beltrão et al., 1991). Novos métodos vêm sendo propostos, aliado ao desenvolvimento e aprimoramento da capacidade computacional e avanços matemáticos, em especial na álgebra linear. Como exemplo temos os trabalhos de Faddeev et al., (2007) e Wu et al., (2004).

Esse trabalho realiza uma análise comparativa entre a uti- lização do método de decomposição em valores singulares SVD (Zhao B. B. et al., 2011), da técnica de análise da principal componente PCA (Rao C. R., 1964) e do polinômio robusto PR (Leão et. all., 1996), na estimativa dos campos regional-residual extraído a partir de dados gravimétricos sintéticos. Estes dados foram produzidos por uma combinação de efeitos de corpos adjacentes com geometria simples, e que simulam a presença de fontes rasas e profundas, com e sem ruído. A partir do entendimento dessas análises, implementamos uma forma de interpretação conjunta visando a exploração da boa capacidade de filtragem do ruído, apresentada pelo PCA, aliada a boa capacidade de separação demostrada com o uso do SVD. Os algoritmos foram implementados em linguagem Python, linguagem de programação de alto nível, interpretada, orientada objeto e de tipagem dinâmica e forte, cujas bibliotecas estão abertas ao público (matplotlib, numpy, Sklearn e Pandas foram as utilizadas na produção desse trabalho).

\section{Metodologia}

A aplicação do método de decomposição em valores singulares (SVD) na filtragem do dado gravimétrico, produz a separação do dado em suas diferentes componentes. Essas são agrupadas em três partes, cujas contribuições serão interpretadas como campos regional, residual e ruído. Portanto, considerando que $\mathrm{X}$ é a matriz $\mathrm{MxN}$ que contém os valores do campo gravimétrico calculado ou observado, dada por:

$$
\mathbf{X}=\mathbf{U S V}^{\mathbf{T}},
$$

onde $\mathbf{S}$ é uma matriz não quadrada com $\sigma_{i}$ (valores singulares) elementos na diagonal principal, organizados em ordem decrescente de magnitude, contendo todos os elementos fora da diagonal principal preenchidos por zeros. Os valores de $\sigma_{i}$ são obtidos como sendo $\sigma_{i}=\sqrt{\lambda_{i}}$ de $C=\mathbf{X}^{\mathbf{T}} \mathbf{X}$. $\mathbf{V}^{\mathrm{t}}$ é o conjugado transposto de $\mathbf{V}$, onde $\mathbf{V}$ é uma matriz $\mathrm{N} \times \mathrm{N}$ na qual suas colunas são os autovetores de $C$ e $\mathbf{U}$ uma matriz $\mathrm{MxM}$, que projeta $\mathbf{X}$ nos autovetores de $C$.

Os menores valores de $\sigma_{i}$ são atribuídos ao ruído. Para melhor determinar o valor de corte entre o sinal e ruído é possível se fazer uma analise gráfica dos valores de $\sigma$ pelos respectivos índices $i$, conhecido como espectro singular (Figura 1). A partir da Figura 1 e após a escolha desse valor de corte, poderemos zerar os valores de $\sigma$ além do índice selecionado e assim gerar um sinal livre de ruído (Clifford G. D., 2005). Essa escolha pode ainda permitir separar o regional do residual e neste caso, selecionamos os maiores valores de $\sigma$, faixa correspondente ao regional, assim como repetir o processo anterior só que zerando os valores até esse índice, o que permite a extração do 
residual. Essa decomposição permitirá a diferenciação de fontes independentes num sinal sem ruído.

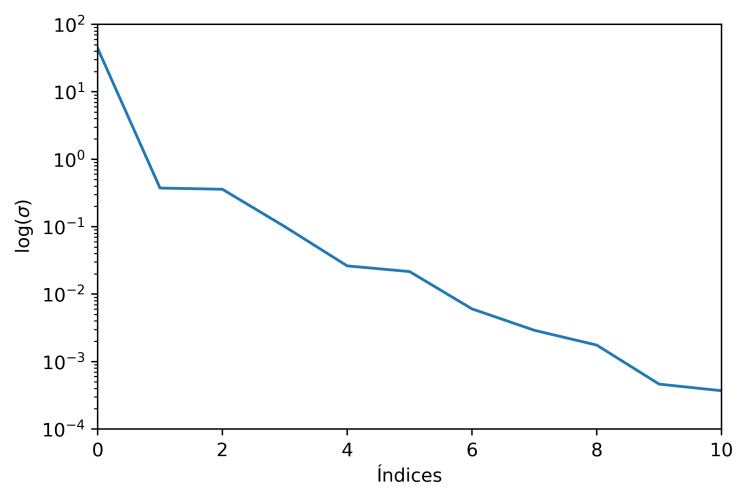

Figura 1: Espetro dos dez maiores valores singulares, que é utilizado na filtragem do dado sem ruído.

A análise de componentes principais (PCA) permite, através de uma transformação ortogonal, converter o dado em um conjunto de valores de variáveis linearmente não correlacionados (componentes principais). Ela é desenvolvida de forma que o primeiro componente $\left(y_{1}\right)$ possua a maior variância possível, e os seguintes tenha a máxima variância, com a necessidade da ortogonalidade entre eles:

$$
y_{i}=v_{i}^{T} X(i=1,2, \ldots, N),
$$

onde $y_{i}$ são as componentes principais; $v_{i}^{T}$ é a transposta de $v_{i}$ que é a direção do componente e $\mathbf{X}$ é o dado observado. A obtenção de $v_{i}$ é feita a partir da técnica de SVD, que corresponde aos autovetores de $C=\mathbf{X}^{\mathbf{T}} \mathbf{X}$. A aplicação do PCA é muito parecida com a do SVD, porém a escolha dos índices de corte para separar regional, residual e ruído foi empírica, através da plotagem e interpretação dos mapas filtrados. O residual é obtido pela subtração da componente principal do dado original, caso essa metodologia não seja seguida ocorrerá uma grande distorção da amplitude na anomalia.

Para a separação regional-residual foi também utilizado o método do polinômio robusto (Leão et. all., 1996), um método amplamente utilizado e que irá servir como técnica referência na comparação dos resultados obtidos a partir do PCA e SVD. No método polinomial, considera-se que a estimativa do campo regional é feita por um polinômio de grau $n$ e este ajuste é realizado pelo método dos mínimos quadrados, impondo que a soma dos resíduos seja mínima, podendo gerar pseudo-anomalias com sinal positivo e/ou negativo. Beltrão et al.(1989), propõe um ajuste polinomial onde aos resíduos são atribuídos pesos para retirar essas pseudo-anomalias.

\subsection{Modelo gravimétrico sintético}

As técnicas descritas foram aplicadas a um modelo sintético mostrado na Figura 2 e que produziu o mapa de anomalia Bouguer apresentado na Figura 3. Acrescentamos também diferentes níveis de ruído aleatório (em porcentagem) no valor da anomalia Bouguer calculada pelo modelo sintético citado. O mapa cuja a razão sinal/ruído é de aproximada- mente 4,98 é mostrado na Figura 8 e foi utilizado em todo o desenvolvimento desse trabalho.

Tabela 1: Posição e características da esferas.

\begin{tabular}{|c|l|l|l|l|}
\hline Esfera & $\begin{array}{c}\mathrm{X} \\
(\mathrm{km})\end{array}$ & $\begin{array}{c}\mathrm{y} \\
(\mathrm{km})\end{array}$ & $\begin{array}{c}\text { Profundi- } \\
\text { dade } \\
(\mathrm{m})\end{array}$ & $\begin{array}{c}\text { Densi- } \\
\text { dade } \\
\left(\frac{K G}{m^{3}}\right)\end{array}$ \\
\hline \hline 1 & 2 & 8 & 1200 & 3.2 \\
\hline 2 & 5 & 5 & 800 & 3.0 \\
\hline 3 & 7 & 2 & 1200 & 3.0 \\
\hline
\end{tabular}

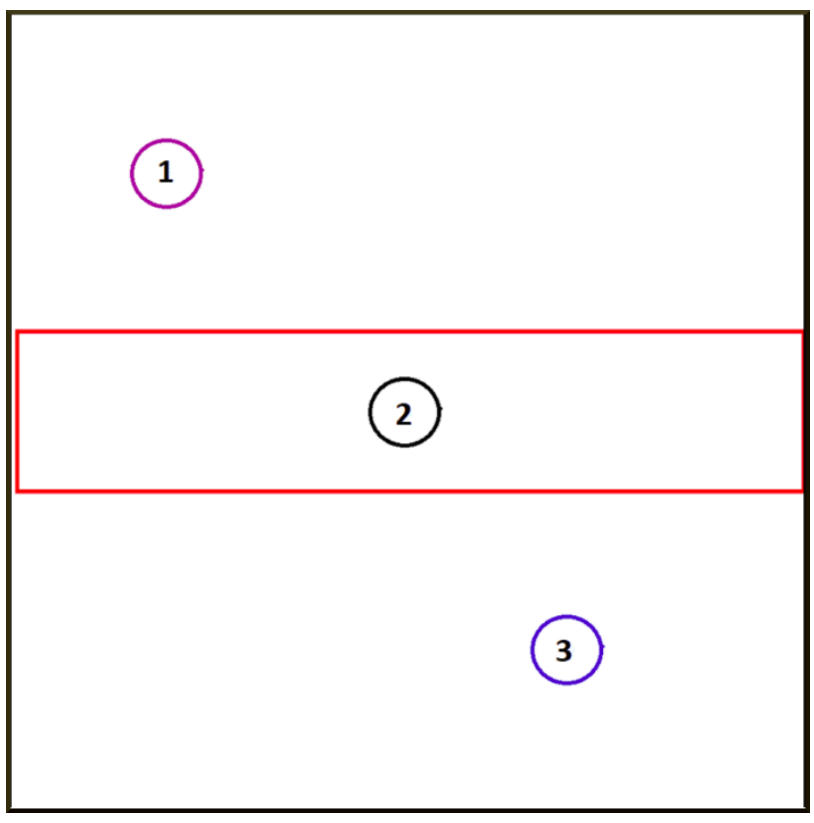

Figura 2: Esquema de posicionamento dos corpos (fora de escala).

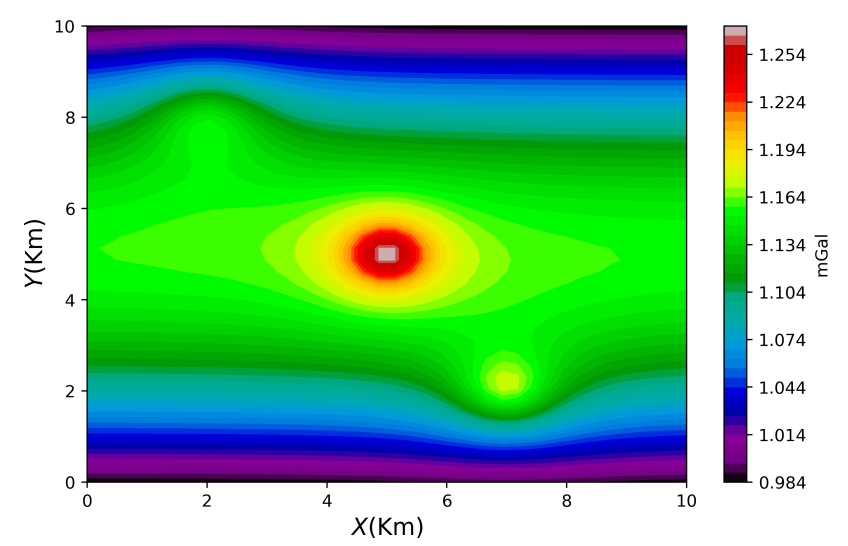

Figura 3: Anomalia Bouguer sem ruído, obtido a partir do modelo numérico apontado pela 2 .

O modelo sintético mostrado na Figura 2, é composto por três esferas de mesmas características geométricas (todas possuem 100 metros de raio) colocadas em diferentes posições e profundidades (ver Tabela 1) e um cilindro de 350 metros de raio com seu eixo paralelo a direção $x$, com profundidade de 12 quilômetros. As esferas buscam simular o efeito residual de corpos em diferentes profundidades, 
enquanto que o efeito regional foi simulado a partir do cilindro, de modo que sua influência no campo gravimétrico total gerado seja bastante suave.

\subsection{Estimativa do campo regional e re- sidual}

Inicialmente testamos a eficiência na separação regionalresidual dos três métodos descritos aos dados da anomalia sintética sem ruído (Figura 3). As técnicas de SVD e PCA geraram mapas residuais, conforme, respectivamente, as Figuras 5 e 6 , de estilos parecidos com boa resolução para os corpos causadores destas anomalias, porém podemos notar a presença de artefatos espúrios que podem representar a adição de um conteúdo de ruído não aleatório, de forma espelhada e de baixa amplitude com pouca coerência. $O$ PCA e SVD geraram uma grande redução na magnitude da anomalia residual, como esperado ao zerar algumas componentes, e o PCA nos limites do modelo, apresentou feições anômalas que podemos ser entendidas como efeitos de borda.

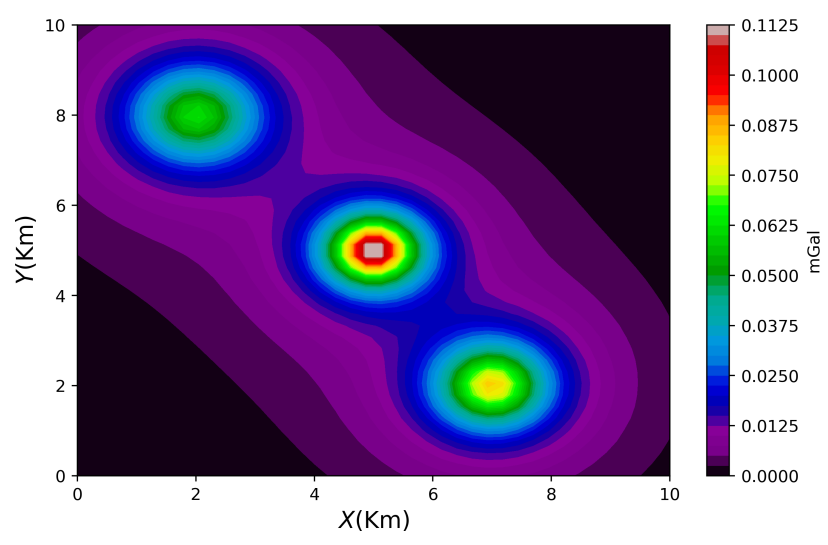

Figura 4: Anomalia residual sem ruído.

Na aplicação do PR (Figura 7) esses artefatos não são observados, fazendo com que as contribuições regional-residual fossem bem distinguíveis, produzindo uma interpretação mais simples e menos susceptíveis a erros. Como o PR foi utilizado para a estimativa do campo regional, o residual foi obtido após a subtração entre o campo gravimétrico total e o campo regional estimado pelo ajuste robusto.

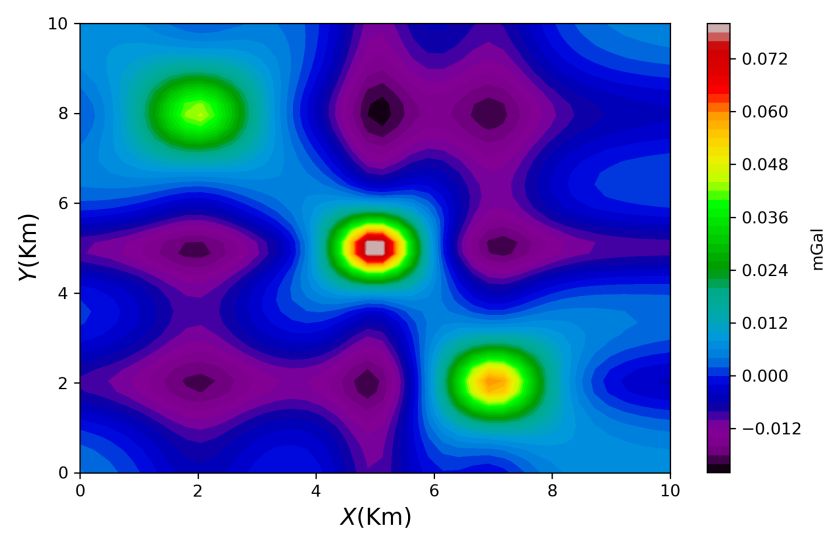

Figura 5: Anomalia residual sem ruído, utilizando SVD.

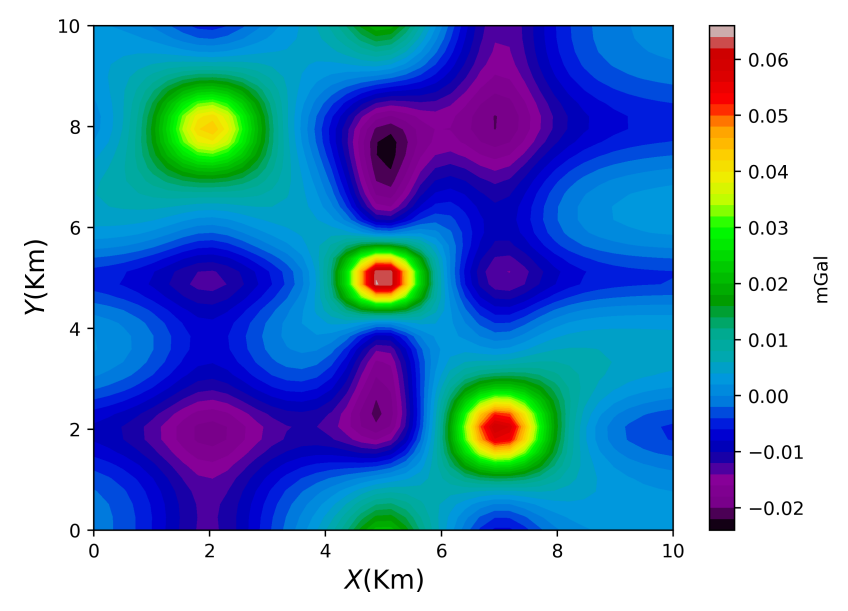

Figura 6: Anomalia residual sem ruído, utilizando PCA.

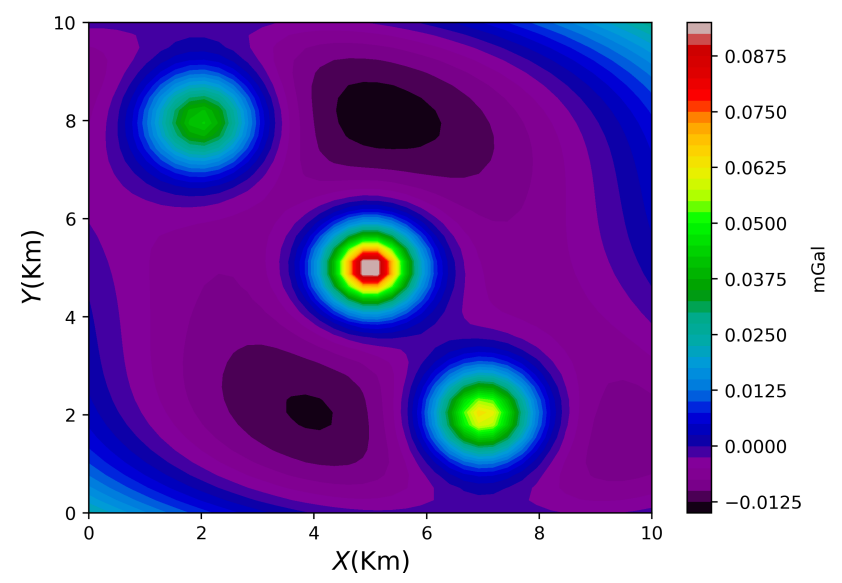

Figura 7: Anomalia residual sem ruído, utilizando PR.

Ao analisar esses mapas é valido ressaltar que no SVD e PCA foi utilizado uma técnica de interpretação diferente da usualmente utilizada no PR, na qual se busca interpretar anomalias mais coerentes em intensidade, já que apesar de criar artefatos lineares essas apresentam magnitudes inferiores a anomalia desejada.

Apesar dessa perda na magnitude ao se analisar o erro dentre as três técnicas utilizadas o SVD foi a de menor valor e o PR a de maior.

\subsection{Atenuação de ruído}

A Figura 8 apresenta os dados sintéticos com adição de ruído. Isso nos permitiu avaliar a capacidade de filtragem do ruído destes métodos, assim como seu efeito sobre o procedimento de separação do regional-residual. As técnicas SVD e PCA também foram aplicadas para a extração do ruído nesses dados e cujos mapas resultantes são mostrados nas Figuras 9 e 10. Ela revelou que a filtragem de ruído usando SVD é mais efetiva que a produzida pelo PCA.

As Figuras demonstram a efetiva atenuação do ruído usando ambos os métodos, e que as anomalias resultantes apresentam formas e dimensões pouco parecidas com o mapa sem ruído (Figura 4). Ambos os casos produziram mapas muito parecidos mas o SVD recuperou melhor a influencia 


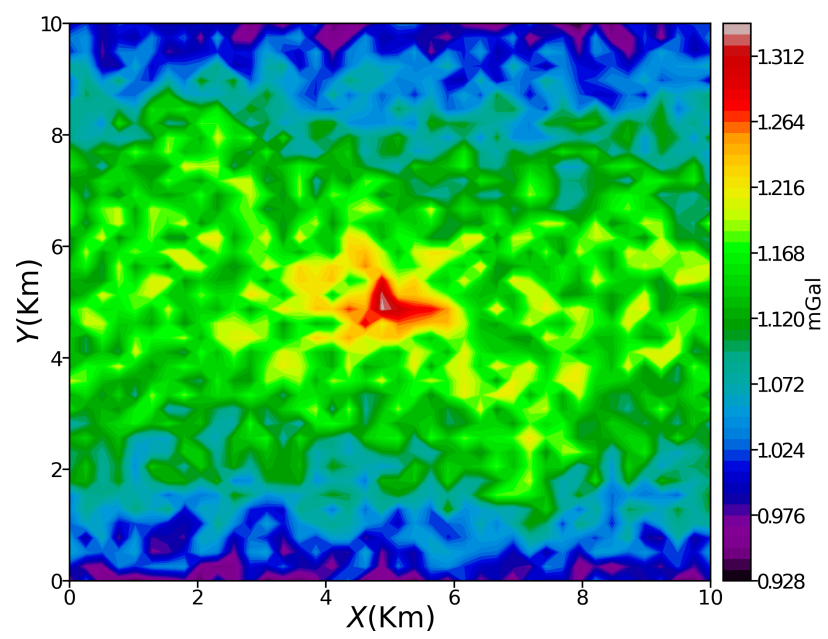

Figura 8: Anomalia Bouguer com 10\% de ruído.

suave do campo regional e obteve o menor valor do erro em relação ao esperado (Figura 3). Em ambos os casos houve um ganho na magnitude das anomalias em relações ao valor esperado, fato especialmente notado no caso da aplicação SVD, em que para todos os níveis de ruído aplicados, o mapa apresentou valores mais distantes da realidade com o aumento do ruído. Observa-se também a presença de ruídos não aleatórios nestes mapas, artefatos oriundos das metodologias aplicadas. Nos dois casos se abre a possibilidade de eliminação do ruído aleatório, o que não poderia ser feita com a aplicação do PR diretamente aos dados ruidosos.

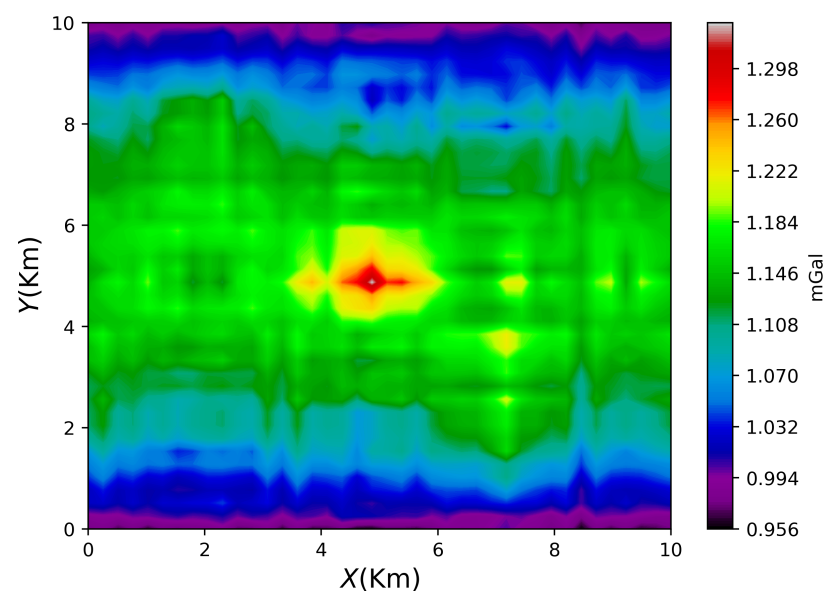

Figura 9: Mapa anomalia Bouguer (10\% de ruído) com filtragem de ruído SVD.

Realizamos também uma associação entre os métodos, buscando aproveitar o melhor de cada um deles. Nessa associação, a técnica de SVD foi utilizada para a eliminação do ruído e para a separação regional-residual as técnicas PCA, SVD e PR, visando a obtenção do residual.

\subsection{Separação residual associada}

A Figura 11 resultou da separação usando a técnica de PCA, o que localizou bem a esfera central, apesar da perda de geometria e produzir um alongamento na direção $x$, possivelmente, em virtude da presença do cilindro. Porém foi possível detectar a existência de dois outros corpos,

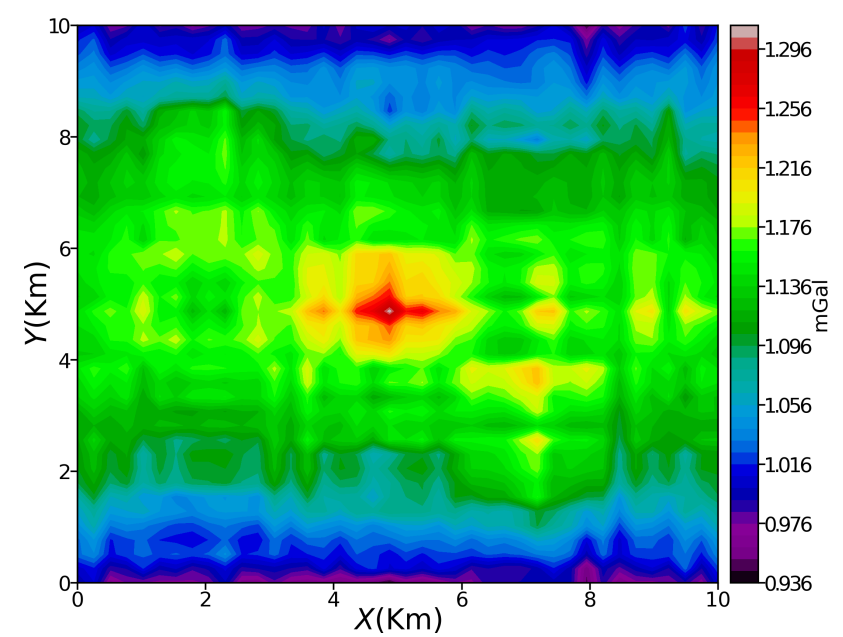

Figura 10: Mapa anomalia Bouguer (10\% de ruído) com filtragem de ruído PCA.

mas com completa perda de sua geometria. A contribuição das esferas 1 e 3 não foram bem ressaltadas, sendo vista apenas como uma espécie de altos gravimétricos de segunda ordem. Anomalias de magnitude intermediária sofreram um alongamento mais severo. Observa-se que ocorreu perda da amplitude da anomalia para menos, mas a relação de magnitude entre as esferas foi preservada, fazendo com que a interpretação dessa separação seja mais qualitativa, em termos de localização e geometria, do que quantitativa, em termos de densidade e profundidade.

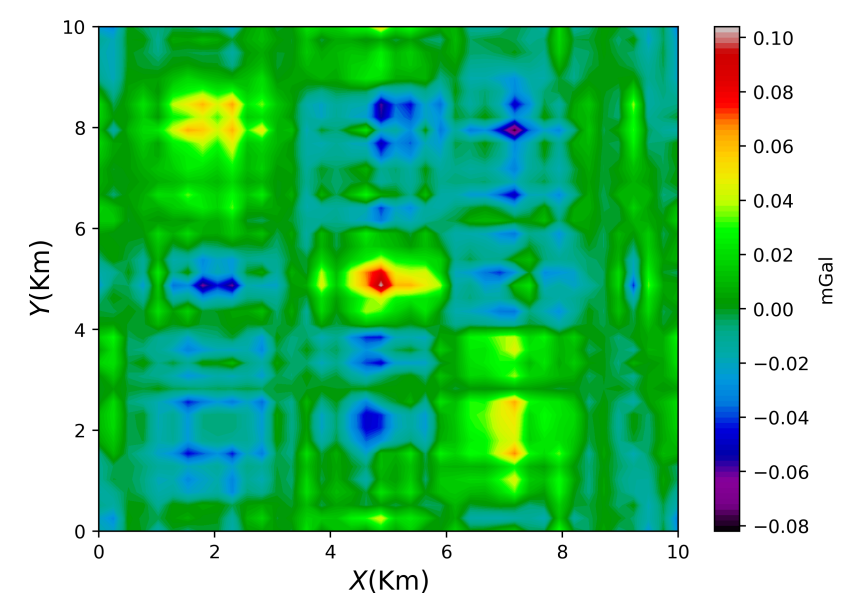

Figura 11: Filtragem SVD - Mapa residual gerado utilizando PCA.

No caso da separação usando SVD (figura 12), os resultados foram mais significativos, pois ambas esferas (1 e 3) foram mais ressaltadas, em relação ao resto do mapa, comparativamente ao PCA. Porém, somente a anomalia resultante da esfera 2 (central) tive sua forma recuperada de alguma forma. A análise da magnitude das anomalias levou ao caso inverso a do PCA, o valor obtido é maior que o ideal, fato surpreendente já que é esperado pela forma como essas técnicas funcionam que se perca amplitude no sinal ao zerar as componentes usadas para representar o modelo (somatório das mesmas). Assim essa técnica permite, preferencialmente, uma interpretação qualitativa muito precisa quanto a existencia de corpos e suas profundidades porem a utilização de forma quantitativa se mostrou imprecisa. 


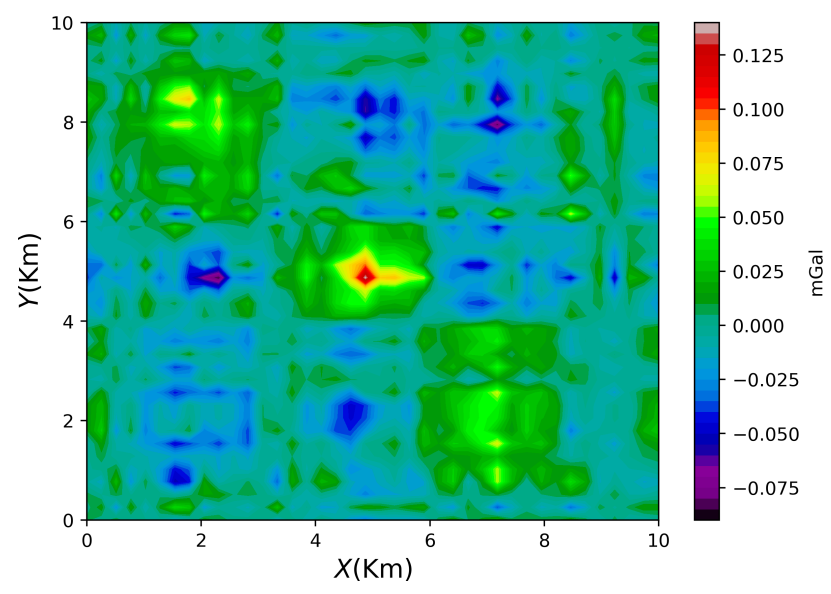

Figura 12: Filtragem SVD - Mapa residual gerado utilizando SVD.

Os resultados utilizando o polinômio robusto (Figura 13), para fins comparativos, mostrou que seus resultados foram pouco relevantes.

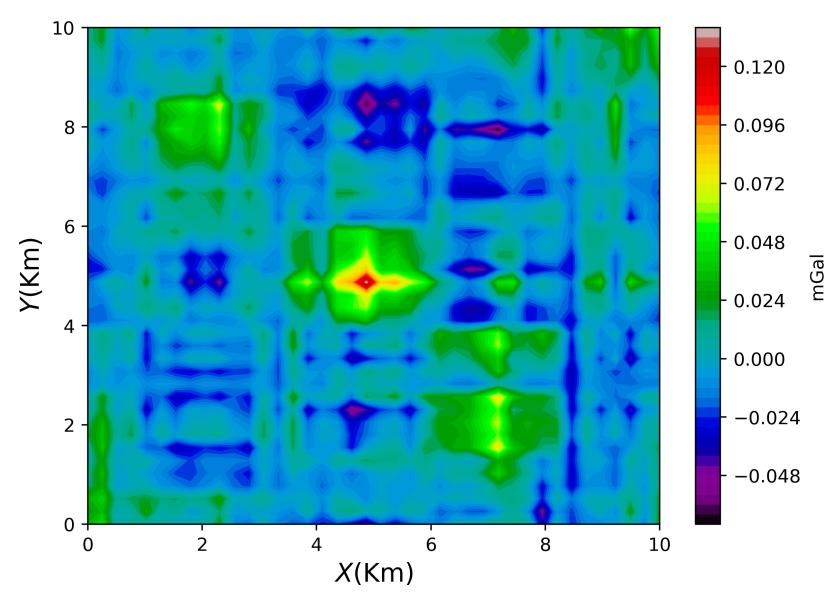

Figura 13: Filtragem PCA - Mapa residual gerado utilizando polinômio robusto.

Ao se analisar o erro quadrático médio de cada uma das associações foi notado que o menor valor foi alcançado com a associação do SVD para atenuar o ruído com o PCA para separar o residual.

\section{Discussão e Conclusões}

Após a analise dos resultados obtidos, foi possível notar que a filtragens utilizando as técnicas de componentes (SVD e PCA), criaram uma forte tendência em todo o dado, linearização no sentido y para as anomalias de alta magnitude, e no sentido $x$ para as de magnitudes médias. Porém, a técnica SVD se demostrou efetiva na atenuação do ruído. Também foi notado a parcial ou completa perda de resolução em termos de geometria das anomalias, porém o local e centro foram mantidos sobre os corpos e ainda se pôde diferenciar as fontes profundas das rasas, através da interpretação de cada autoimagem gerada pelas técnicas (quanto mais profundo maior o índice da autoimagem).

A linearização notada nos dados resultantes da atenuação de ruído pelas técnicas SVD e PCA, foi interpretada como fruto da eliminação de ruído devido aos métodos de componentes. Assim a técnica de SVD pôde ter resultados melhores, em especial do ponto de vista geométrico dos corpos, se for utilizado outro método de eliminação/atenuação do ruído.

A recuperação do residual para dados com media razão sinal/ruído (com $10 \%$ de ruído) demostrou que as técnicas são excelentes em determinar a profundidade e posição dos corpos, mas a recuperação da amplitude não foi notada, já que tanto o SVD e PCA reduziram a amplitude das anomalias sem perder, em ambos os casos, a relação entre elas. Porém, isso não impossibilita a percepção, associando conhecimentos geológicos e das autoimagens, sobre qual anomalia é a mais profunda, o que pode servir como informação inicial para modelagens futuras.

\section{Referências}

[1] J. F. Beltrão, J. B. C. Silva, and J. C. Costa. Robust polynomial fitting methods for regional gravity estimation. Geophysics, 56:80-89, 1991.

[2] Richard J Blakely. Potential theory in gravity and magnetic applications. Cambridge University Press, 1996.

[3] G. D. Clifford. Singular value decomposition \& independent component analysis for blind source separation. HST582J/6.555 J/16.456 J Biomedical Signal and Image Processing Spring, 2005.

[4] Dmitrī Konstantinovich Faddeev and Vera Nikolaevna Faddeeva. Computational methods of linear algebra. 2007.

[5] Shib Sankar Ganguli and VP Dimri. Interpretation of gravity data using eigenimage with indian case study: A svd approach. Journal of Applied Geophysics, 95:23-35, 2013.

[6] B. H. Jacobsen. A case for upward continuation as a standard separation filter for potential-field maps. Geophysics, 52:390-398, 1987.

[7] J. W. D. Leão, P. T. L. Menezes, and J. B. C. Beltrão, J. F.and Silva. Gravity inversion of basement relief constrained by the knowledge of depth at isolated points. Geophysics, 61:1702-1714, 1996.

[8] K. L. Mickus and W. C. James. Regional gravity studies in south-eastern california, western arizona and southern nevada. J. Geophys Res., 96(12):333350, 1991.

[9] C. R. Rao. The use and interpretation of principal component analysis in applied research. Sankhya Series, 26:329-358, 1964.

[10] Jon Shlens. A tutorial on principal component analysis derivation. Discussion and Singular Value Decomposition, 25, 2003.

[11] Chun-Guo Wu, Y Liang, Y Sun, C Zhou, and Y Lu. On the equivalence of svd and pca. CHINESE JOURNAL OF COMPUTERS-CHINESE EDITION-, 27(2):286288, 2004.

[12] BB Zhao and YQ Chen. Singular value decomposition (svd) for extraction of gravity anomaly associated with gold mineralization in tongshi gold field, western 
shandong uplifted block, eastern china. Nonlinear

Processes in Geophysics, 18(1):103, 2011. 